\title{
A faster distributed approximation scheme for the connected dominating set problem for growth- bounded graphs
}

\section{Report}

Author(s):

Gfeller, Beat; Vicari, Elias

Publication date:

2011

Permanent link:

https://doi.org/10.3929/ethz-a-006783117

Rights / license:

In Copyright - Non-Commercial Use Permitted

Originally published in:

Technical Report / ETH Zurich, Department of Computer Science 540 


\title{
A Faster Distributed Approximation Scheme for the Connected Dominating Set Problem for Growth-Bounded Graphs
}

\author{
ETH Technical Report 540 - Revised Version* \\ Beat Gfeller ${ }^{\star \star}$ and Elias Vicari ${ }^{\star \star \star}$ \\ Institute of Theoretical Computer Science, \\ ETH Zurich, Switzerland \\ \{gfeller,vicariel\}@inf.ethz.ch
}

\begin{abstract}
We present a distributed algorithm for finding a $(1+\varepsilon)$ approximation of a Minimum Connected Dominating Set in the class of Growth-Bounded graphs, which includes Unit Disk graphs. In addition, the computed Connected Dominating Set guarantees a constant stretch factor on the length of a shortest path with respect to the original graph and induces a subgraph of constant degree. The nodes do not require any positioning or distance information.

The algorithm runs in $O\left(T_{\mathrm{MIS}}+1 / \varepsilon^{O(1)} \cdot \log ^{*} n\right)$ synchronous rounds, where $T_{\text {MIS }}$ is the time for computing a Maximal Independent Set (MIS) in the network graph. Using the fastest known deterministic algorithm for computing a MIS, the total running time is $O\left(\left(\log \Delta+1 / \varepsilon^{O(1)}\right) \cdot \log ^{*} n\right)$, where $\Delta$ is the maximum degree of the network graph. If one allows randomization, the running time reduces to $O\left(\left(\log \log n+1 / \varepsilon^{O(1)}\right) \cdot \log ^{*} n\right)$ rounds.
\end{abstract}

Keywords. Connected Dominating Set, Growth-Bounded Graphs, Distributed Approximation Scheme, Distributed Algorithms.

\section{Introduction}

Wireless sensor networks are used in an increasing number of current applied research projects, in a variety of applications ranging from agricultural management targeted at Indian farmers [18] to body area networks for human activity recognition [11]. To assist these developments, it is becoming ever more important to find solutions to the fundamental difficulties in employing such networks.

\footnotetext{
* The first version of this technical report was submitted as a ETH Technical Report in November 2006.

** This author gratefully acknowledges the support of the Swiss SBF under contract no. C05.0047 within COST-295 (DYNAMO) of the European Union.

$\star \star \star$ This author is partially supported by the National Competence Center in Research on Mobile Information and Communication Systems NCCR-MICS, a center supported by the Swiss National Science Foundation under grant number 5005 - 67322.
} 
A wireless ad hoc network typically consists of a large number of nodes, each having wireless communication capability, modest computational power, and a battery with limited capacity. Two nodes can communicate directly if they are within mutual communication range, and forward messages via intermediate nodes to talk to distant nodes.

In contrast to wired networks, where usually a dedicated backbone infrastructure with high-throughput capabilities is available for long-distance routing, ad hoc networks do not have any a priori means to manage the routing and scheduling of messages. Another specialty of wireless ad hoc networks is mobility of the nodes, which may continuously cause changes in the topology.

In the absence of an organized routing scheme, simple flooding (i.e., the first time a message is received from any neighbor, it is forwarded to all other neighbors) could be used to transmit messages. However, this is very wasteful in terms of energy and causes interference problems if many nodes transmit a message at the same time. To organize routing more cleverly, a virtual backbone can be computed, i.e., a subset of the nodes which participate in multi-hop routing. In this fashion, even when messages are still forwarded by flooding inside the virtual backbone, the energy savings are significant.

When modelling the network as a graph, the most widely used concept for defining a backbone is the Connected Dominating Set (CDS). A CDS is a subset $S$ of the nodes of a graph $G$ that induces a connected subgraph of $G$, such that every node that is not in $S$ has a neighbor in $S$.

Usually, the energy savings are higher if the number of nodes in the CDS is small. However, computing a Minimum CDS is NP-hard even on Unit Disk Graphs [4], and would require global information. Considering the highly dynamic nature of ad hoc networks, it is important that the CDS can be computed locally within a short time; a linear running time in the diameter of the network (as required to obtain global information) is clearly inappropriate. Moreover, a minimum CDS lacks some desirable properties which we demand from an efficient backbone. For instance, routing a message from a node $v$ to node a $w$ should not need many more intermediate hops than a shortest path in the original network. The maximum ratio over all vertex pairs between these two hop-distances is called the stretch factor. The stretch factor of a MCDS can be as bad as linear in the number of nodes (for instance, consider a ring network), and one would like to prevent this effect. Another desirable property is that nodes have constant degree in the CDS-induced graph, which might help to address interference issues. For these reasons, a natural trade-off is to find a CDS with only near-optimal size but fulfilling the aforementioned properties.

Related Work. The concept of a virtual backbone (in analogy to backbones in wired networks) was introduced in [6]. Since then, the construction of small connected dominating sets in Unit Disk Graphs (UDGs) has been intensively studied. A recent overview can be found in [2]. For the centralized setting with given coordinates of the nodes (which are embedded in the Euclidean plane), a polynomial-time approximation scheme (PTAS) was proposed in [3] (approxima- 
tion schemes for related problems like the Minimum Dominating Set Problem in UDGs were given in [10]). The approach of [5] (as well as our own approach) yields a PTAS that does not require coordinate information about the nodes.

However, many of the first distributed algorithms either did not guarantee a good approximation ratio in the worst case, or had a linear running time (see [2]). The first approach achieving a constant approximation ratio in polylogarithmic time was [19]. Alzoubi et al. [1] were the first to provide an algorithm for computing a CDS with low stretch and low degree, which was coined well-connected CDS in [19]. Recently, Czygrinow et al. [5] presented a distributed algorithm with running time $O\left(1 / \varepsilon^{6} \cdot \log (1 / \varepsilon) \cdot \log ^{3} n\right)$ achieving the approximation ratio $1+\varepsilon$ for any $\varepsilon>0$, but without proving any stretch or degree bounds.

In general graphs, the best known distributed algorithm for the MCDS problem has a polylogarithmic running time and achieves an approximation ratio of $O(\log \Delta)$, where $\Delta$ is the maximum degree of the network [7]. This approximation ratio is asymptotically optimal unless all problems in NP can be solved by deterministic algorithms with running time $n^{O(\log \log n)}[8]$.

Our Contribution. In this paper we present a distributed approximation scheme for the problem of finding a Minimum Connected Dominating Set in the class of growth-bounded graphs, which includes Unit Disk Graphs. An important feature of our algorithm is that the only information required by the nodes is the set of their direct neighbors. Distances between them or even coordinate information are not required.

The algorithm computes a well-connected $(1+\varepsilon)$-approximation of a Connected Dominating Set, for any $\varepsilon>0$. This takes $O\left(T_{\mathrm{MIS}}+1 / \varepsilon^{O(1)} \cdot \log ^{*} n\right)$ rounds of synchronous computation, where $T_{\mathrm{MIS}}$ is the number of rounds needed to compute a Maximal Independent Set (MIS). Currently, the fastest deterministic distributed algorithm for computing a MIS in growth-bounded graphs is due to Kuhn et al. [12] and runs in $O\left(\log \Delta \cdot \log ^{*} n\right)$ time. Using randomization, a MIS can even be computed in $O\left(\log \log n \cdot \log ^{*} n\right)$ time in growth-bounded graphs [9]. Thus, we improve on the running time for computing a $(1+\varepsilon)$-approximate CDS, while adding the guarantee that the computed CDS has constant stretch, constant degree, and therefore a linear number of edges.

The algorithm we propose builds substantially on the approach of Nieberg et al. [16] for computing a $(1+\varepsilon)$-approximate Minimum Dominating Set (for growth-bounded graphs): In a nutshell, their solution partitions the graph into clusters of appropriate radius, computes an optimal DS on each of these, and takes the union of these sets to yield a DS for the graph. Employing the same idea, we cluster the graph and compute an optimal CDS for each cluster. However, we let the clusters overlap such that the union of the small CDS solutions forms a connected DS of the graph. We prove the approximation ratio of the CDS by adapting the proof from [16], which requires an additional non-trivial step, i.e., Lemma 7. In the proof of Lemma 7, ideas related to [5] are used. In addition, we prove the (well-)connectedness of the computed set, which has no equivalent in $[16,5]$. In order to turn our centralized approximation scheme for CDS into a distributed algorithm, we follow the lines of [13]. 
Intriguingly, such a relatively simple modification of known techniques yields a distributed approximation scheme which runs substantially faster than the previously known solution [5], and additionally guarantees well-connectedness.

\section{Definitions}

Network model. We model a wireless network as a growth-bounded graph, a class which includes Unit Disk graphs. The precise definition follows in the next subsection. Each node has a unique identifier and knows which nodes are within its transmission range. When we say that a distributed algorithm computes a CDS, we mean that each node knows after executing its code whether it is part of the CDS or not. Assuming that a MAC layer for direct communication of neighbors has already been established, we do not consider collisions or other transmission failures, and we furthermore assume a synchronous network.

Terminology. Let $G=(V, E)$ be an undirected connected graph. For $V^{\prime} \subseteq V$, we denote by $G\left[V^{\prime}\right]$ the subgraph of $G$ induced by $V^{\prime}$ : the vertex-set of $G\left[V^{\prime}\right]$ is $V^{\prime}$ and the edge-set consists of the edges of $G$ with both endpoints in $V^{\prime}$.

The distance $d(v, w)$ between two vertices $v, w \in V$ is the number of edges (or hops) that must be traversed to go from $v$ to $w$ on a shortest path. Similarly, we define the distance between two sets $A, B \subseteq V$ as the distance of two closest nodes $a \in A$ and $b \in B$, i.e., $d(A, B)=\min \{d(a, b) \mid a \in A, b \in B\}$. Two sets $S, T \subseteq V$ are called 2-separated ${ }^{1}$ if and only if $d(S, T) \geq 3$.

The neighborhood $N(v)$ of a vertex $v \in V$ is defined as the set all vertices $w \in V$ with $d(v, w) \leq 1$ (including $v$ ). The neighborhood of a set $S \subseteq V$ is defined as $N(S):=\bigcup_{s \in S} N(s)$. Likewise, the $i$-neighborhood of a set of vertices $S$ is defined recursively as $N_{0}(S):=S$, and $N_{i}(S):=N_{i-1}(N(S))$ for $i \geq 1$. The reduced neighborhood $\Gamma_{j}\left(v, V^{\prime}\right)$ is defined for all $j \geq 0$ as $N_{j}(v)$ on the graph induced by the set $V^{\prime} \subseteq V$.

A maximal independent set (MIS) $M$ for a given graph $G=(V, E)$ is a subset $M \subseteq V$ such that for every $v, w \in M$ we have $v \notin N(w)$ (independence) and furthermore no superset $M^{\prime} \supset M$ with the latter property exists (maximality). A maximum independent set (MaxIS) is a MIS of largest size. For a subset $S \subseteq$ $V, \operatorname{Max} I S(S)$ is a maximum independent set on the induced subgraph $G[S]$. A dominating set (DS) $D$ for a given graph $G=(V, E)$ is a subset $D \subseteq V$ such that for every $v \in V$ there is a $w \in D$ such that $v \in N(w)$. Note that a MIS is always a DS. A connected dominating set (CDS) for a given graph $G=(V, E)$ is a dominating set $M$ with the additional requirement that the nodes in $M$ induce a connected subgraph of $G$. A minimum (or optimal) connected dominating set (MCDS) is a CDS of smallest possible cardinality, and the minimum connected dominating set problem is the problem of providing such a MCDS for a given graph. We define the function $\mathcal{C}(A)$, where $A \subseteq V$ is a subset of all nodes, as

\footnotetext{
${ }^{1}$ We use the term 2-separation because any shortest path between $S$ and $T$ contains two nodes outside $S \cup T$.
} 
follows: $\mathcal{C}(A) \subseteq N(A)$ is a minimum connected set of nodes that dominates all nodes in $A$, under the condition that only nodes in $N(A)$ are used. It is crucial that this set may contain nodes from $V \backslash A$.

We are interested in a special class of graphs, called growth-bounded graphs.

Definition 1. A graph $G$ is growth-bounded if there is a polynomial bounding function $f(r)$ such that for each node $v \in V$, the size of any MIS in the neighborhood $N_{r}(v)$ is at most $f(r), \forall r \geq 0$.

A subclass of growth-bounded graphs are Unit Disk Graphs, which are often used to model wireless communication networks. A graph $G=(V, E)$ is a Unit Disk Graph if it can be represented by placing a point $p_{v}$ for each node $v \in V$ on the Euclidean plane $\mathbb{R}^{2}$, such that an edge $(u, v) \in E$ exists if and only if the distance $\left\|p_{v}-p_{u}\right\|_{2}$ is at most 1 .

Unit Disk Graphs are growth-bounded with a bounding function $f(r) \in$ $O\left(r^{2}\right)$, which is easily proved as follows: consider any node $v$ and any independent set $I \subseteq N_{r}(v)$, for a $r \geq 0$. The corresponding points of two (independent) nodes in $I$ must have distance greater than 1 . Thus each point corresponding to a node in $I$ exclusively occupies a disk of radius $1 / 2$ with an area of $(1 / 2)^{2} \pi$. As all these disks must lie inside a circle of radius $r+1 / 2$, it follows that $|I| \leq$ $\frac{(r+1 / 2)^{2} \pi}{(1 / 2)^{2} \pi}=4 r^{2}+4 r+1$. Furthermore, most other graph classes used to model wireless ad-hoc networks such as Quasi Unit Disk Graphs, Cover Area Graphs and other intersection graphs are growth-bounded [14,17].

To end this section, we introduce two properties of optimal connected dominating sets in growth-bounded graphs, which we use in our approach.

Lemma 1. For any growth-bounded graph $G=(V, E)$ with bounding function $f$, there is a polynomial $p(r) \leq 3 \cdot f(r)$ such that for any $v \in V$, it holds $\left|\mathcal{C}\left(N_{r}(v)\right)\right| \leq$ $p(r), \forall r>0$.

Proof. Let $f(r)$ be the polynomial bounding function of the growth-bounded graph $G$. Consider a maximal independent set $I$ of $N_{r}(v)$ (for a fixed $r \geq 0$ ) and set $Q:=I$. Let $k=|I|$ be the number of components of $G[I]$. We proceed by induction over $k$. If $k=1, G[Q]$ is connected and the claim follows. Otherwise, since $I$ is also a dominating set of $N_{r}(v)$, we can find two connected components $A, B \subseteq Q$ such that $d(A, B) \leq 3$. By adding to $Q$ the vertices of a shortest path between them, we decrease the number of components by at least one and we increase $|Q|$ by at most two. We proceed inductively until $Q$ induces a connected graph. Since $k=|I|$, we get $\left|\mathcal{C}\left(N_{r}(v)\right)\right| \leq|Q| \leq|I|+2|I| \leq 3 f(r)$. As this holds for every $r>0$, the claim is proved.

Lemma 2. Let $G=(V, E)$ be a growth-bounded graph with bounding function $f$, and choose a $S \subseteq V$ that induces a connected subgraph. Then for any MIS $M$ of $S$, it holds: $|M| \leq f(1) \cdot|\mathcal{C}(S)|$.

Proof. As $\mathcal{C}(S)$ dominates $S$, each node in $M$ must have a neighbor in $\mathcal{C}(S)$ (or be in $\mathcal{C}(S)$ itself). But by definition, at most $f(1)$ nodes in $M$ can have the same neighbor in $\mathcal{C}(S)$, so the claim follows. 


\section{Finding a Small Connected Dominating Set}

In the following, we describe a (sequential) procedure to construct for each $\varepsilon>0$ a connected dominating set of size at most $(1+\varepsilon)$ times the minimum. This procedure can be executed efficiently in a centralized way, and thus leads to a PTAS. Moreover, in Section 4, we show that the same procedure can be implemented efficiently in a distributed way, using the same technique as in [13].

The CDS is constructed by computing optimal connected dominating sets for small parts of the graph, and taking the union of these CDSs. We will construct the small CDSs such that their union leads to a connected set, as required. Each small CDS is an optimal solution of a small cluster specified as follows: choose any node $v \in V$, and consider the $r$-neighborhood of $v$ for increasing values of $r=0,1,2, \ldots$ until we find a large enough $r^{*}$ such that

$$
\left|\operatorname{Max} \operatorname{IS}\left(N\left(\Gamma_{r^{*}}(v)\right) \backslash \Gamma_{r^{*}}(v)\right)\right| \leq \varepsilon \cdot\left|\operatorname{MaxIS}\left(\Gamma_{r^{*}}(v)\right)\right|
$$

holds. We call this operation an expansion of $v$. As we show in Lemma 4 below, $r^{*}$ is bounded by a function in $O(1 / \varepsilon \cdot \log (1 / \varepsilon))$ depending solely on $\varepsilon$ for any class of growth-bounded graphs.

Our algorithm for finding a CDS for $G$ proceeds as follows: starting with an empty set $D$, it chooses any node $v_{1} \in V$ of $G$, finds a corresponding $r_{1}^{*}$ such that Inequality 1 holds, and adds $\mathcal{C}\left(\Gamma_{r_{1}^{*}+4}\left(v_{1}, V\right)\right)$ to the current solution $D$. After that, it removes all nodes in $\Gamma_{r_{1}^{*}+2}\left(v_{1}, V\right)$ from the graph $G$ and we denote the set of remaining nodes by $V^{\prime}$. Note that here we do not remove all nodes that are dominated by the current solution $D$ from the graph. This is an important difference to the approach in [16]. As we will show, this modification guarantees that the final solution will be a connected dominating set.

In the reduced graph, the algorithm chooses another node $v_{2} \in V^{\prime}$, considers growing neighborhoods of $v_{2}$, until a $r_{2}^{*}$ satisfying Inequality 1 is found. Note that the bounding function $f$ of the original graph is still valid for the reduced graph, because any set which is independent in the reduced graph is also independent in the original graph. Furthermore, recall that $\mathcal{C}\left(\Gamma_{r_{2}^{*}}\left(v_{2}, V^{\prime}\right)\right)$ and $\mathcal{C}\left(\Gamma_{r_{2}^{*}+4}\left(v_{2}, V^{\prime}\right)\right)$ may contain some nodes from the original graph $G$ as dominators which are outside $V^{\prime}$ because they are already dominated. Then, $\mathcal{C}\left(\Gamma_{r_{2}^{*}+4}\left(v_{2}, V^{\prime}\right)\right)$ is added to the current solution $D$, and all nodes in $\Gamma_{r_{2}^{*}+2}\left(v_{2}, V^{\prime}\right)$ are removed from the graph, just as before. Then, this procedure is repeated until all nodes have been removed from the graph.

The algorithm is described formally in Algorithm 1. Since the set of remaining nodes should always be clear from the context, we omit the second argument of $\Gamma(\cdot, \cdot)$ in the rest of the paper.

For proving Lemma 4, we need the following fact.

Lemma 3. Consider any class of growth-bounded graphs with bounding function $f$, and a graph $G=(V, E)$ of this class. Then for any $r \geq 1$ and $v \in V$ it holds:

$$
\left|\operatorname{Max} I S\left(N\left(\Gamma_{r}(v)\right) \backslash \Gamma_{r}(v)\right)\right| \leq f(2) \cdot\left|\operatorname{MaxIS}\left(\Gamma_{r}(v) \backslash \Gamma_{r-1}(v)\right)\right|
$$




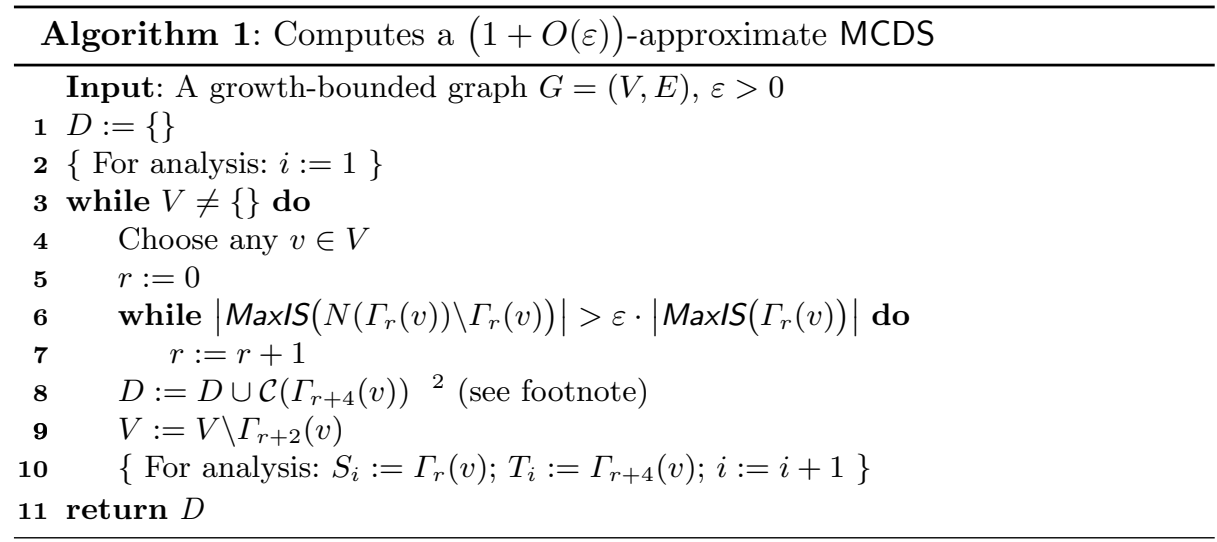

Proof. Clearly, each node in $\operatorname{MaxIS}\left(N\left(\Gamma_{r}(v)\right) \backslash \Gamma_{r}(v)\right)$ has a neighbor in $\Gamma_{r}(v)$ in $G$. As $\operatorname{MaxIS}\left(\Gamma_{r}(v) \backslash \Gamma_{r-1}(v)\right)$ is a dominating set of $\Gamma_{r}(v) \backslash \Gamma_{r-1}(v)$, each node in $\operatorname{Max} \operatorname{IS}\left(N\left(\Gamma_{r}(v)\right) \backslash \Gamma_{r}(v)\right)$ has a node in $\operatorname{MaxIS}\left(\Gamma_{r}(v) \backslash \Gamma_{r-1}(v)\right)$ within distance at most two. However, the number of nodes in $\operatorname{MaxIS}\left(N\left(\Gamma_{r}(v) \backslash \Gamma_{r}(v)\right)\right.$ that lie within two hops of the same node in $\operatorname{MaxIS}\left(\Gamma_{r}(v) \backslash \Gamma_{r-1}(v)\right)$ can be at most $f(2)$, as otherwise these nodes could not be mutually independent.

Lemma 4. Consider any class of growth-bounded graphs with bounding function $f$. Then, for any $\varepsilon>0$, there is a $R_{f}^{*}(\varepsilon)=O(1 / \varepsilon \cdot \log (1 / \varepsilon))$ such that for each graph $G$ of this class, and each node $v$ of $G$, it holds

$$
\left|\operatorname{Max} I S\left(N\left(\Gamma_{r^{*}}(v)\right) \backslash \Gamma_{r^{*}}(v)\right)\right| \leq \varepsilon \cdot\left|\operatorname{Max} I S\left(\Gamma_{r^{*}}(v)\right)\right|
$$

for some $r^{*} \leq R_{f}^{*}$.

Proof. Fix an $\varepsilon>0$ and assume in contradiction that no such $R_{f}^{*}$ exists. This implies that for arbitrarily large values $r^{\prime}$, there is a graph in the class such that for some node $v,\left|\operatorname{MaxIS}\left(N\left(\Gamma_{r^{\prime}}(v)\right) \backslash \Gamma_{r^{\prime}}(v)\right)\right|>\varepsilon \cdot\left|\operatorname{MaxIS}\left(\Gamma_{r^{\prime}}(v)\right)\right|$ holds for all $0 \leq r \leq r^{\prime}$. Consider such a value $r^{\prime} \geq 2$. From

$$
\left|\operatorname{Max} I S\left(N\left(\Gamma_{r^{\prime}}(v)\right) \backslash \Gamma_{r}^{\prime}(v)\right)\right|>\varepsilon \cdot\left|\operatorname{Max} I S\left(\Gamma_{r^{\prime}}(v)\right)\right| \geq \varepsilon \cdot\left|\operatorname{MaxIS}\left(\Gamma_{r^{\prime}-2}(v)\right)\right|
$$

and Lemma 3, we have

$$
\left|\operatorname{MaxIS}\left(\Gamma_{r^{\prime}}(v) \backslash \Gamma_{r^{\prime}-1}(v)\right)\right|>\bar{\varepsilon} \cdot\left|\operatorname{MaxIS}\left(\Gamma_{r^{\prime}-2}(v)\right)\right|, \quad \text { for } \bar{\varepsilon}=\varepsilon / f(2) .
$$

Hence, for all $r: 2 \leq r \leq r^{\prime}$ we have

$$
\begin{aligned}
\left|\operatorname{MaxIS}\left(\Gamma_{r}(v)\right)\right| & \geq\left|\operatorname{MaxIS}\left(\Gamma_{r}(v) \backslash \Gamma_{r-1}(v)\right)\right|+\left|\operatorname{MaxIS}\left(\Gamma_{r-2}(v)\right)\right| \\
& >(1+\bar{\varepsilon}) \cdot\left|\operatorname{MaxIS}\left(\Gamma_{r-2}(v)\right)\right| .
\end{aligned}
$$

\footnotetext{
${ }^{2}$ Recall that $\mathcal{C}\left(\Gamma_{r+4}(v)\right)$ represents the optimal CDS of $\Gamma_{r+4}(v)$.
} 
Assume for the moment that $r^{\prime}$ is an even number. Then we have

$$
\begin{aligned}
\left|\operatorname{MaxIS}\left(\Gamma_{r^{\prime}}(v)\right)\right| & >(1+\bar{\varepsilon}) \cdot\left|\operatorname{MaxIS}\left(\Gamma_{r^{\prime}-2}(v)\right)\right|>(1+\bar{\varepsilon})^{2} \cdot\left|\operatorname{MaxIS}\left(\Gamma_{r^{\prime}-4}(v)\right)\right| \\
& >\ldots>(1+\bar{\varepsilon})^{\frac{r^{\prime}}{2}} \cdot\left|\operatorname{MaxIS}\left(\Gamma_{0}(v)\right)\right|=(1+\bar{\varepsilon})^{\frac{r^{\prime}}{2}}
\end{aligned}
$$

Since $\left|\operatorname{MaxIS}\left(\Gamma_{r^{\prime}}(v)\right)\right|$ grows only polynomially in $r^{\prime}$ (Lemma 1), but the term $(1+\bar{\varepsilon})^{\frac{r^{\prime}}{2}}$ grows exponentially in $r^{\prime}$ (note that $(1+\bar{\varepsilon})^{1 / 2}>1$ ), the above inequality will be violated for some large enough $r^{\prime}$, which is a contradiction. If $r^{\prime}$ is odd, the same reasoning can be applied.

The claimed bound on $R_{f}^{*}$ follows easily from the inequality $(1+\bar{\varepsilon})^{1 / \bar{\varepsilon}}>e-1$, for $\bar{\varepsilon}$ small enough.

It is clear that Algorithm 1 terminates, and that $D$ then contains a dominating set, because only dominated nodes are removed from the graph.

Let $S_{1}, S_{2}, \ldots, S_{k}$ and $T_{1}, T_{2}, \ldots, T_{k}$ be the sets $\Gamma_{r}\left(v_{i}\right)$ and $\Gamma_{r+4}\left(v_{i}\right)$ respectively as chosen in each iteration of the outer while-loop of Algorithm 1. We now show that the computed solution $D$, which consists of the union of the $\mathcal{C}\left(T_{i}\right)$, forms a connected subgraph of $G$.

Lemma 5. The union $D:=\bigcup_{i=1}^{k} \mathcal{C}\left(T_{i}\right)$ induces a connected subgraph of $G$.

Proof. First, we show that any two nodes $a, b \in D$ with distance $d(a, b)=2$ in $G$ are part of the same connected component in (the subgraph induced by) $D$. To that end, consider any node $w \in V$ and its neighbors $N(w)$ (see the left part of Figure 1). Let $s$ be the first node among $N(w)$ (including $w$ ) that is removed in line 9 of Algorithm 1. When $s$ is removed in the $i$-th iteration, it holds that $s \in \Gamma_{r_{i}^{*}+2}\left(v_{i}\right)$ and so all nodes in $N(w)$ are in $T_{i}=\Gamma_{r_{i}^{*}+4}\left(v_{i}\right)$, whereby $v_{1}, v_{2}, \ldots$ represent the centers of the expansions. Hence they are dominated by $\mathcal{C}\left(\Gamma_{r_{i}^{*}+4}\left(v_{i}\right)\right)$. Therefore, any pair of nodes in $D \cap N(w)$ is connected by a path of length at most $p\left(R^{*}\right)+1$ hops consisting only of nodes in $D$ (recall $p$ from Lemma 1).

Second, consider any pair $u, v \notin D$ of nodes adjacent in $G$ (see the right part of Figure 1). We show that there must exist nodes $u^{\prime} \in N(u) \cap D$ and $v^{\prime} \in N(v) \cap D$ such that $u^{\prime}$ and $v^{\prime}$ are connected by a path of length at most $p\left(R^{*}\right)-1$ consisting only of nodes in $D$. To that end, assume w.l.o.g. that $u$ is removed (line 9) before or at the same time as $v$. When $u$ is removed in the $j$-th iteration (i.e., $\left.u \in \Gamma_{r_{j}^{*}+2}\left(v_{j}\right)\right)$, also $v$ is dominated by $\mathcal{C}\left(\Gamma_{r_{j}^{*}+4}\left(v_{j}\right)\right)$. The second claim follows. Combining this with the first claim, we have that any two nodes $a, b \in D$ with distance $d(a, b)=3$ in $G$ are connected by a path of length at most $3 p\left(R^{*}\right)+1$ consisting only of nodes in $D$.

These two facts together imply that $D$ induces a connected subgraph. Indeed, if $D$ were disconnected, then the shortest path in $G$ between two closest components of $D$ would consist solely of nodes in $V \backslash D$. However, such a path cannot be of length two or three by the above facts, and not longer either, because then $D$ would not dominate all the nodes. 

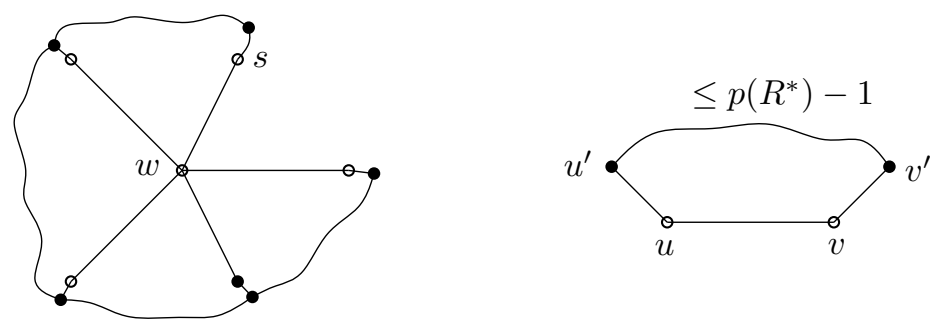

Fig. 1. Illustration of Lemma 5. The filled dots represent nodes that have already joined $D$. The other nodes are represented by empty dots.

Now that we have shown that the set $D$ computed by Algorithm 1 is a connected dominating set, we prove that its size is at most $1+\varepsilon$ times larger than the optimum. To this end, we need two lemmas.

Lemma 6. Let $\varepsilon>0$ and $r>0$ be such that the following inequality is fulfilled:

$$
\left|\operatorname{Max} I S\left(N\left(\Gamma_{r}(v)\right) \backslash \Gamma_{r}(v)\right)\right| \leq \varepsilon \cdot\left|\operatorname{MaxIS}\left(\Gamma_{r}(v)\right)\right| .
$$

Then, for $\varepsilon^{\prime}:=\varepsilon \cdot(3 f(4)+3) \cdot f(1)$, it follows:

$$
\left|\mathcal{C}\left(\Gamma_{r+4}(v)\right)\right| \leq\left(1+\varepsilon^{\prime}\right) \cdot\left|\mathcal{C}\left(\Gamma_{r}(v)\right)\right| .
$$

Proof. We show how to extend $\mathcal{C}\left(\Gamma_{r}(v)\right)$ to a connected dominating set of $\Gamma_{r+4}(v)$ by adding only relatively few nodes so that the claim follows. Let $M$ be a maximal independent set of $\Gamma_{r+1}(v) \backslash \Gamma_{r}(v)$. Any node in $\Gamma_{r+4}(v)$ lies within 3 hops of some node in $\Gamma_{r+1}(v) \backslash \Gamma_{r}(v)$, and thus within 4 hops of some node in $M$. Thus, all nodes in $\Gamma_{r+4}(v)$ are dominated if we add to our solution the set $\mathcal{C}\left(\Gamma_{4}(w)\right)$ for each $w \in M$ (note that $\left|\mathcal{C}\left(\Gamma_{4}(w)\right)\right| \leq p(4) \leq 3 f(4)$ ). In order to connect these sets to $\mathcal{C}\left(\Gamma_{r}(v)\right)$, we need to add at most 3 additional nodes for each $w \in M$. Thus in total, we obtain a connected dominating set of $\Gamma_{r+4}(v)$ of size at most $\left|\mathcal{C}\left(\Gamma_{r}(v)\right)\right|+(3 f(4)+3) \cdot\left|\operatorname{Max} \mathrm{IS}\left(\Gamma_{r+1}(v) \backslash \Gamma_{r}(v)\right)\right| \leq\left|\mathcal{C}\left(\Gamma_{r}(v)\right)\right|+(3 f(4)+$ $3) \cdot\left|\operatorname{Max} I S\left(N\left(\Gamma_{r}(v)\right) \backslash \Gamma_{r}(v)\right)\right| \leq\left|\mathcal{C}\left(\Gamma_{r}(v)\right)\right|+\varepsilon \cdot(3 f(4)+3) \cdot\left|\operatorname{Max} I S\left(\Gamma_{r}(v)\right)\right| \leq$ $\left|\mathcal{C}\left(\Gamma_{r}(v)\right)\right|+\varepsilon \cdot(3 f(4)+3) \cdot f(1) \cdot\left|\mathcal{C}\left(\Gamma_{r}(v)\right)\right|$, using Lemma 2 for the last inequality.

Let $V^{*}$ be the set of nodes chosen as centers $v$ for the growing neighborhoods in the algorithm. As for each $v \in V^{*}, \Gamma_{r+2}(v)$ is removed from the graph before choosing a new node, the collection $\left\{S_{1}, S_{2}, \ldots, S_{k}\right\}$ consists of 2-separated sets. We have the following lower bound for the size of an optimal CDS for $G$.

Lemma 7. Let $S_{1}, S_{2}, \ldots, S_{k}$ be the collection of 2-separated sets in $G=(V, E)$ computed by Algorithm 1 and $\varepsilon^{\prime \prime}:=2 f(1) \varepsilon$. Then,

$$
\left(1+\varepsilon^{\prime \prime}\right) \cdot|\mathcal{C}(V)| \geq\left|\bigcup_{i=1}^{k} \mathcal{C}\left(S_{i}\right)\right| .
$$


Proof. Since the $S_{i}$ are 2-separated, the sets $N\left(S_{i}\right)$ are disjoint, so the sets $\mathcal{C}(V) \cap N\left(S_{i}\right)$ are disjoint, too. Furthermore, as $\mathcal{C}(V)$ must dominate all nodes of $G$, including those in $S_{i}$, the set $\mathcal{C}(V) \cap N\left(S_{i}\right)$ must dominate all nodes in $S_{i}$. To complete the proof, we now show that $\left|\mathcal{C}\left(S_{i}\right)\right| \leq\left(1+\varepsilon^{\prime \prime}\right) \cdot\left|\mathcal{C}(V) \cap N\left(S_{i}\right)\right|$ for all $i$, and thus, $|\mathcal{C}(V)| \geq \sum_{i=1}^{k}\left|\mathcal{C}(V) \cap N\left(S_{i}\right)\right| \geq \frac{1}{1+\varepsilon^{\prime \prime}} \cdot \sum_{i=1}^{k}\left|\mathcal{C}\left(S_{i}\right)\right|$.

To that end, we add some nodes to $\mathcal{C}(V) \cap N\left(S_{i}\right)$ in order to obtain a connected set which dominates $S_{i}$. Let $x$ be the number of connected components in $\mathcal{C}(V) \cap$ $N\left(S_{i}\right)$ and suppose that $x \geq 2$, otherwise $\mathcal{C}(V) \cap N\left(S_{i}\right)$ is connected and hence $\left|\mathcal{C}\left(S_{i}\right)\right| \leq\left(1+\varepsilon^{\prime \prime}\right) \cdot\left|\mathcal{C}(V) \cap N\left(S_{i}\right)\right|$ is trivial. Then the individual connected components of $\mathcal{C}(V) \cap N\left(S_{i}\right)$ can be connected by adding at most $2 x$ nodes (see the proof of Lemma 1). Note that each connected component of $\mathcal{C}(V) \cap N\left(S_{i}\right)$ must contain one node from $N\left(\Gamma_{r}(v)\right) \backslash \Gamma_{r}(v)$ to ensure the global connectivity of the solution. Thus, by choosing one such node for each connected component of $\mathcal{C}(V) \cap N\left(S_{i}\right)$, we obtain an independent set of size $x$. Therefore, we have $x \leq\left|\operatorname{MaxIS}\left(N\left(S_{i}\right) \backslash S_{i}\right)\right|$. By construction of the $S_{i},\left|\operatorname{MaxIS}\left(N\left(S_{i}\right) \backslash S_{i}\right)\right| \leq \varepsilon$. $|\operatorname{Max}| \mathrm{S}\left(S_{i}\right) \mid$, and by Lemma $2,\left|\mathcal{C}\left(S_{i}\right)\right| \geq\left|\operatorname{MaxIS}\left(S_{i}\right)\right| / f(1)$.

So we can obtain a connected set that dominates $S_{i}$ by adding to $\mathcal{C}(V) \cap N\left(S_{i}\right)$ at most $2 \varepsilon f(1) \cdot\left|\mathcal{C}\left(S_{i}\right)\right|$ nodes. The claim now follows by choosing $\varepsilon^{\prime \prime}=2 f(1) \varepsilon$.

Theorem 1. The set $D$ computed by Algorithm 1 is a $(1+O(\varepsilon))$-approximation for the connected dominating set problem.

Proof. Let $\left\{S_{1}, S_{2}, \ldots, S_{k}\right\}$ and $\left\{T_{1}, T_{2}, \ldots, T_{k}\right\}$ be as defined in Algorithm 1. By Lemma 6 , it holds that $\left|\mathcal{C}\left(T_{i}\right)\right| \leq\left(1+\varepsilon^{\prime}\right) \cdot\left|\mathcal{C}\left(S_{i}\right)\right|$ for all $i=1, \ldots, k$, and $D=\bigcup_{i=1}^{k} \mathcal{C}\left(T_{i}\right)$. Hence we have

$$
\begin{gathered}
|D|=\left|\bigcup_{i=1}^{k} \mathcal{C}\left(T_{i}\right)\right| \leq \sum_{i=1}^{k}\left|\mathcal{C}\left(T_{i}\right)\right| \leq\left(1+\varepsilon^{\prime}\right) \cdot \sum_{i=1}^{k}\left|\mathcal{C}\left(S_{i}\right)\right| \\
=\left(1+\varepsilon^{\prime}\right) \cdot\left|\bigcup_{i=1}^{k} \mathcal{C}\left(S_{i}\right)\right| \leq\left(1+\varepsilon^{\prime}\right)\left(1+\varepsilon^{\prime \prime}\right) \cdot|\mathcal{C}(V)|,
\end{gathered}
$$

where the last inequality follows from Lemma 7 .

We now shortly discuss how Algorithm 1 can be implemented in a centralized fashion to obtain a PTAS. Most steps of the algorithm can be trivially computed efficiently. The crucial part is the computation of the maximum independent sets $\operatorname{Max} \operatorname{IS}\left(N\left(\Gamma_{r}(v)\right) \backslash \Gamma_{r}(v)\right)$ and $\operatorname{MaxIS}\left(\Gamma_{r}(v)\right)$, and of $\mathcal{C}\left(\Gamma_{r^{*}+4}(v)\right)$. Note that $r$ is bounded by the constant $R^{*}$ for any fixed $\varepsilon$, so from the growth-bounded property we know that the size of the MaxIS is bounded by a constant. Thus, by enumerating all node subsets of cardinality at most $f(r)$, and selecting the largest of those which is both independent and maximal, a maximum independent set is found in polynomial time. Since the considered subsets have only constant size, independence and maximality of the subsets can be checked in constant time. The same arguments apply to the computation of $\mathcal{C}\left(\Gamma_{r^{*}+4}(v)\right)$, due to Lemma 1. Hence, Algorithm 1 has polynomial time complexity for any fixed $\varepsilon>0$, but exponential time complexity in $1 / \varepsilon$. 


\section{A Fast Distributed Approximation Scheme}

The main goal of this paper is to provide a fast distributed algorithm that computes a $(1+\varepsilon)$-approximation for the MCDS problem in growth-bounded graphs. The algorithm we describe here is an adaptation from [13], adjusted to computing a CDS instead of a DS.

A naive distributed implementation would be that in each round all nodes which have the highest ID within their $2 R^{*}+9$-hop neighborhood are expanded concurrently. However, this approach requires a linear number of rounds in the worst case, because there can be a linear waiting chain of nodes. The observation that every expansion affects only neighbors within a small radius leads to a more efficient algorithm: expansions of nodes with sufficient mutual distances can be scheduled concurrently. Roughly, this can be achieved by computing a MIS of $G$, and then coloring this MIS with few colors such that two nodes with the same color are distant enough. The coloring is achieved using a clustergraph $\bar{G}$ of radius $c=c(\varepsilon)$ with centers $W \subseteq V: \bar{G}=(\bar{V}, \bar{E})$, where $\bar{V}:=W$ and for every $u, v \in W(u, v) \in \bar{E}$ if and only if $\bar{d}(u, v) \leq c$. Note that if $W$ is an independent set and $G$ is growth-bounded, $\bar{G}$ has a maximum degree of $\Delta_{\bar{G}}=O(f(c))$. Hence using a MIS of $G$ to construct a clustergraph $\bar{G}$ of radius $c, \bar{G}$ can be colored with $O\left(\Delta_{\bar{G}}^{2}\right)$ colors in $O\left(c \cdot \log ^{*} n\right)$ time [15]. Note that a communication round in the cluster graph costs $O(c)$ rounds in the original graph. The coloring is then used to schedule the expansion of neighbors of MIS-nodes. We choose $c=2 R^{*}+11$ for reasons becoming apparent in the proof of Lemma 9. A more detailed description is given in Algorithm 2.

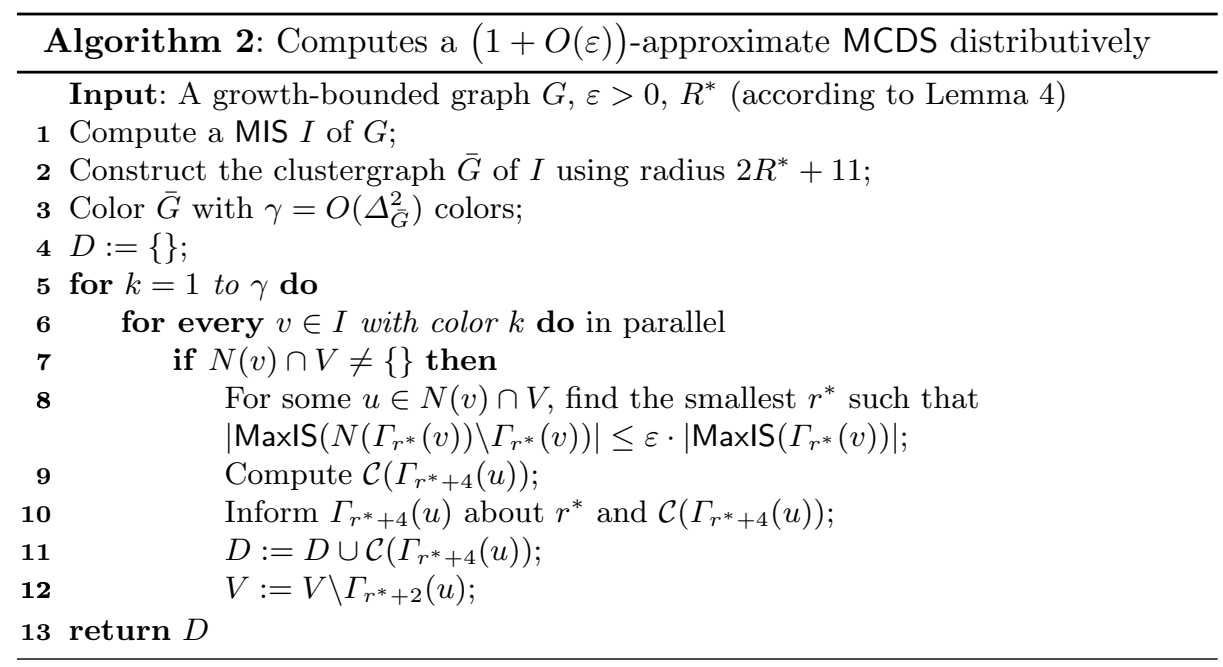

Lemma 8. Algorithm 2 terminates in $O\left(T_{M I S}+1 / \varepsilon^{O(1)} \cdot \log ^{*} n\right)$ time.

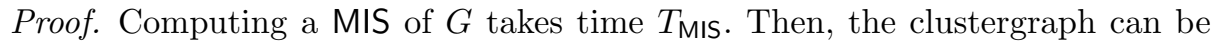
constructed in constant time, as its edges only span at most distance $2 R^{*}+11=$ 
$O(1 / \varepsilon \cdot \log (1 / \varepsilon))$. Furthermore, $\bar{G}$ can be colored with $O\left(\Delta_{\bar{G}}^{2}\right)=O\left(f^{2}\left(R^{*}\right)\right)$ colors in $O\left(R^{*} \cdot \log ^{*} n\right)$ time using the well-known algorithm of [15].

The outer for-loop is executed $O\left(\Delta_{\bar{G}}^{2}\right)$ times. Inside the for-loop, the number of different MaxIS that each node $u$ (as in line 8) must compute is $2 r^{*}=O\left(R^{*}\right)=$ $O(1 / \varepsilon \cdot \log (1 / \varepsilon))$. For computing each MaxIS and MCDS for a neighborhood of radius $r, u$ collects all information about $\Gamma_{r}(u)$ and then computes the set locally. As $r \leq R^{*}$, all steps in lines 8 to 12 can be executed in $O(1 / \varepsilon \cdot \log (1 / \varepsilon))$ time.

Lemma 9. The set $D$ computed by Algorithm 2 is a $(1+O(\varepsilon))$-approximate minimum connected dominating set.

Proof. By construction, any two nodes that are concurrently used for an expansion have distance at least $2 R^{*}+9$, because they are respective neighbors of two MIS nodes of distance at least $2 R^{*}+11$. The radius used by either expansion is at most $R^{*}$, and since each expansion only involves the nodes within a radius of at most $R^{*}+5$, all concurrent expansions would have the same result if they were executed sequentially. Therefore, there exists an execution of the sequential Algorithm 1 which computes the same set $D$ as Algorithm 2. It follows that Algorithm 2 achieves the same approximation ratio as Algorithm 1.

These two lemmas lead to our main theorem.

Theorem 2. For any $\varepsilon>0$, Algorithm 2 computes a $(1+O(\varepsilon))$-approximate minimum connected dominating set in $O\left(T_{M I S}+1 / \varepsilon^{O(1)} \cdot \log ^{*} n\right)$ time.

\section{Well-Connectedness}

The connected dominating set computed by our Algorithm 1 is not only a $(1+$ $\varepsilon)$-approximation of a minimum CDS, but has additional properties which are desirable for its usage as a backbone in a wireless network. Let $G^{\prime}\left(V^{\prime}, E^{\prime}\right)$ be the graph induced by the $\operatorname{CDS}_{\mathcal{A}}$ of the (growth-bounded) graph $G=(V, E)$ computed by Algorithm 1. Then, it holds for any $\varepsilon>0$ :

1. The backbone graph $G^{\prime}$ has maximum degree $O\left(1 / \varepsilon^{O(1)}\right)$, and therefore it has only $O\left(1 / \varepsilon^{O(1)} \cdot\left|V^{\prime}\right|\right)$ edges;

2. using $G^{\prime}$ as a routing backbone guarantees a $O\left(1 / \varepsilon^{O(1)}\right)$ stretch.

We assume in the following that source $s$ and destination $d$ of a routing request are both members of the CDS. If this is not true for either or both of them, then we can easily choose a neighbor inside the CDS as a representative. This will add at most two hops to the routing path, so if the stretch is low for any pair $s, d$ inside the CDS, the stretch of any pair $s, d$ inside $\mathrm{G}$ is also low.

To make the second statement precise, define $\lambda:=\max _{u, v \in V^{\prime}} \frac{d_{G^{\prime}}(u, v)}{d_{G}(u, v)}$ as the hop-stretch of $G^{\prime}$. Furthermore, if $G$ is a UDG and if $D_{G}(u, v)$ denotes the geometric length of a shortest path in $G$, then the geometric stretch of $G^{\prime}$ is $\mu:=\max _{u, v \in V^{\prime}} \frac{D_{G^{\prime}}(u, v)}{D_{G}(u, v)}$. 
Lemma 10. Let $C D S_{\mathcal{A}}$ be the CDS computed by Algorithm 1. The subgraph $G^{\prime}$ of $G$ induced by the nodes in $C D S_{\mathcal{A}}$ has maximum degree $O\left(1 / \varepsilon^{O(1)}\right)$.

Proof. First, note that each partial CDS $T_{i}$ computed by the algorithm covers a subgraph with diameter $O\left(R^{*}\right)$, so according to Lemma $1,\left|T_{i}\right|$ and therefore its degree is at most $O\left(f\left(R^{*}\right)\right)$. Second, each node $u$ of $G$ can only be contained in $O\left(f\left(R^{*}\right)\right)$ many different $T_{i}$, because any expansion that leads to some $T_{j}$ containing $u$ must have as its center a MIS-node in distance at most $R^{*}+2=$ $O(1 / \varepsilon \cdot \log (1 / \varepsilon))$ from $u$ (and there are only $O\left(f\left(R^{*}\right)\right)$ MIS nodes in distance $O\left(R^{*}\right)$ from $\left.v\right)$.

Lemma 11. For any $\varepsilon>0$, the hop-stretch $\gamma$ of $G^{\prime}$ is $O\left(f\left(R^{*}\right)\right)=O\left(1 / \varepsilon^{O(1)}\right)$. Further, if $G$ is a $U D G$, then the geometric stretch $\lambda$ of $G^{\prime}$ is also $O\left(1 / \varepsilon^{O(1)}\right)$.

Proof. Consider any source $s$ and destination $d$ in $V^{\prime}$. Let $\mathcal{P}=\left\langle p_{1}, p_{2}, \ldots, p_{k}\right\rangle$ be the sequence of nodes in a shortest path in $G$ from $s=p_{1}$ to $d=p_{k}$. We define a new path $\mathcal{Q}$ going through nodes $\left\langle q_{1}, q_{2}, \ldots, q_{k}\right\rangle$ as follows: $q_{i}:=p_{i}$ if $p_{i} \in D$. Otherwise, let $q_{i}$ be any node in $D \cap N\left(p_{i}\right)$ (such a node exists because $D$ is dominating). Note that $q_{i} \in D, \forall i: 1 \leq i \leq k$. From the proof of Lemma 5 , we can conclude that between any pair $\left(q_{i}, q_{i+1}\right), \forall i: 1 \leq i \leq k-1$, there is a path in $D$ of length at most $3 p\left(R^{*}\right)+1=O\left(f\left(R^{*}\right)\right)$. Hence there is a path $\mathcal{Q}$ of length $\leq k\left(3 p\left(R^{*}\right)+1\right)=k \cdot O\left(f\left(R^{*}\right)\right)=k \cdot O\left(1 / \varepsilon^{O(1)}\right)$ from $q_{1}$ to $q_{k}$ solely consisting of nodes in $D$.

For the geometric stretch in UDGs, note that in the path $\mathcal{R}:=\left\langle t_{1}=s, t_{2}, \ldots t_{k}=\right.$ $d\rangle$ of shortest geometric length, the outer two of any three consecutive nodes $t_{i}, t_{i+1}, t_{i+2}$ must have distance at least $1:\left\|t_{i}, t_{i+2}\right\|_{2} \geq 1, \forall i \in\{1, \ldots, k-2\}$. So $\mathcal{R}$ with $k$ hops has length at least $(k-1) / 2$. On the other hand, the path with the fewest number of hops (at most $k$ ) has length at most $k$. Since we have shown just before that $G^{\prime}$ includes a path with hop-stretch $\gamma=O\left(1 / \varepsilon^{O(1)}\right)$ between any pair of nodes, it follows that the geometric stretch $\lambda$ of $G^{\prime}$ is at most $2 k \cdot \gamma /(k-1) \leq 4 \gamma=O\left(1 / \varepsilon^{O(1)}\right), \forall k \geq 2$. For $k=1$, the path with fewest hops has length at most $\gamma=O\left(1 / \varepsilon^{O(1)}\right)$, which completes the claim.

Summarizing, we have the following.

Theorem 3. The CDS computed by Algorithm 1 / Algorithm 2 is well-connected.

\section{Conclusion}

The distributed algorithm we presented computes a $(1+\varepsilon)$-approximate Minimum Connected Dominating Set with $O\left(1 / \varepsilon^{O(1)}\right)$ degree and stretch in time $O\left(T_{\text {MIS }}+1 / \varepsilon^{O(1)} \cdot \log ^{*} n\right)$ for growth-bounded graphs. Hence, a faster algorithm for computing a MIS in growth-bounded graphs would directly improve the running time of our solution for fixed $\varepsilon>0$. Clearly, any approach that requires a MIS for constructing a CDS must have a running time of $\Omega\left(\log ^{*} n\right)$ because of the lower bound by Linial [15]. It is an intriguing open question whether a constant approximation for the MCDS problem can be achieved in $o\left(\log ^{*} n\right)$ or even constant time using a fundamentally different approach. 
Acknowledgements We thank an anonymous reviewer of the Ad-Hoc Now 2007 Conference for pointing out an error in our original analysis of the algorithm's approximation ratio.

\section{References}

1. K. M. Alzoubi. Connected dominating set and its induced position-less sparse spanner for mobile ad hoc networks. In Proceedings of the 8th IEEE Intern. Symposium on Computers and Communications (ISCC), pages 209-216, 2003.

2. J. Blum, M. Ding, A. Thaeler, and X. Cheng. Handbook of Combinatorial Optimization, chapter Connected dominating set in sensor networks and MANETs, pages 329-369. Kluwer Academic Publishers, 2004.

3. X. Cheng, X. Huang, D. Li, W. Wu, and D.-Z. Du. A polynomial-time approximation scheme for the minimum-connected dominating set in ad hoc wireless networks. Networks, 42(4):202-208, 2003.

4. B. N. Clark, C. J. Colbourn, and D. S. Johnson. Unit disk graphs. Discrete Mathematics, 86(1-3):165-177, 1990.

5. A. Czygrinow and Michał Hańćkowiak. Distributed approximation algorithms in unit-disk graphs. In 20th Intern. Symposium on Distributed Computing (DISC), volume 4167 of $L N C S$, pages 385-398, 2006.

6. B. Das, R. Sivakumar, and V. Bharghavan. Routing in ad-hoc networks using a virtual backbone. In 6th Intern. Conference on Computer Communications and Networks (IC3N '97), pages 1-20, 1997.

7. D. P. Dubhashi, A. Mei, A. Panconesi, J. Radhakrishnan, and A. Srinivasan. Fast distributed algorithms for (weakly) connected dominating sets and linear-size skeletons. Journal of Computer and System Sciences, 71(4):467-479, 2005.

8. U. Feige. A threshold of $\ln \mathrm{n}$ for approximating set cover. In Proceedings of the 28th Annual ACM Symposium on Theory of Computing (STOC), pages 314-318, 1996.

9. B. Gfeller and E. Vicari. A Randomized Distributed Algorithm for the Maximal Independent Set Problem in Growth-Bounded Graphs. In Proceedings of the 26th Annual ACM SIGACT-SIGOPS Symposium on Principles of Distributed Computing $(P O D C), 2007$.

10. H. B. Hunt III, M. V. Marathe, V. Radhakrishnan, S. S. Ravi, D. J. Rosenkrantz, and R. E. Stearns. NC-approximation schemes for NP- and PSPACE-hard problems for geometric graphs. J. Algorithms, 26(2):238-274, 1998.

11. H. Junker, M. Stäger, G. Tröster, D. Blättler, and O. Salama. Wireless networks in context aware wearable systems. In Proceedings of the 1st European Workshop on Wireless Sensor Networks (EWSN), pages 37-40, 2004.

12. F. Kuhn, T. Moscibroda, T. Nieberg, and R. Wattenhofer. Fast deterministic distributed maximal independent set computation on growth-bounded graphs. In 19th Intern. Symposium on Distributed Computing (DISC), pages 273-287, 2005.

13. F. Kuhn, T. Moscibroda, T. Nieberg, and R. Wattenhofer. Local Approximation Schemes for Ad Hoc and Sensor Networks. In 3rd ACM Joint Workshop on Foundations of Mobile Computing (DIALM-POMC), 2005.

14. F. Kuhn, R. Wattenhofer, and A. Zollinger. Ad-Hoc Networks Beyond Unit Disk Graphs. In 1st ACM Joint Workshop on Foundations of Mobile Computing (DIALM-POMC), 2003.

15. N. Linial. Locality in distributed graph algorithms. SIAM Journal on Computing, 21(1):193-201, 1992. 
16. T. Nieberg and J. L. Hurink. A PTAS for the minimum dominating set problem in unit disk graphs. Memorandum 1732, University of Twente, Enschede, 2004.

17. T. Nieberg and J. L. Hurink. Wireless communication graphs. In Proceedings of DEST Intern. Workshop on Signal Processing for Sensor Networks, ISSNIP'04, pages 367-372, 2004.

18. J. Panchard, S. Rao, T. Prabhakar, H. Jamadagni, and J.-P. Hubaux. COMMONSense Net: Improved Water Management for Resource-Poor Farmers via Sensor Networks. In Intern. Conference on Communication and Information Technologies and Development (ICTD), 2006.

19. S. Parthasarathy and R. Gandhi. Distributed algorithms for coloring and domination in wireless ad hoc networks. In Foundations of Software Technology and Theoretical Computer Science (FSTTCS), pages 447-459, 2004. 